\title{
Gradhiva
}

GRADHIV

Revue d'anthropologie et d'histoire des arts

$7 \mid 2008$

Le possédé spectaculaire

\section{Négocier avec les esprits tromba à Mayotte}

Retour sur le « théâtre vécu » de la possession

Negotiating with tromba spirits in Mayotte. The 'théatre vécu' of possession

revisited

Bertrand Hell

(2) OpenEdition

Journals

Édition électronique

URL: http://journals.openedition.org/gradhiva/1062

DOI : 10.4000/gradhiva. 1062

ISSN : 1760-849X

Éditeur

Musée du quai Branly Jacques Chirac

Édition imprimée

Date de publication : 15 mai 2008

Pagination : $6-23$

ISBN : 978-2-915133-86-8

ISSN : 0764-8928

Référence électronique

Bertrand Hell, « Négocier avec les esprits tromba à Mayotte », Gradhiva [En ligne], 7 | 2008, mis en ligne le 15 mai 2011, consulté le 19 avril 2019. URL : http://journals.openedition.org/gradhiva/1062 ; DOI : 10.4000/gradhiva.1062

(c) musée du quai Branly 


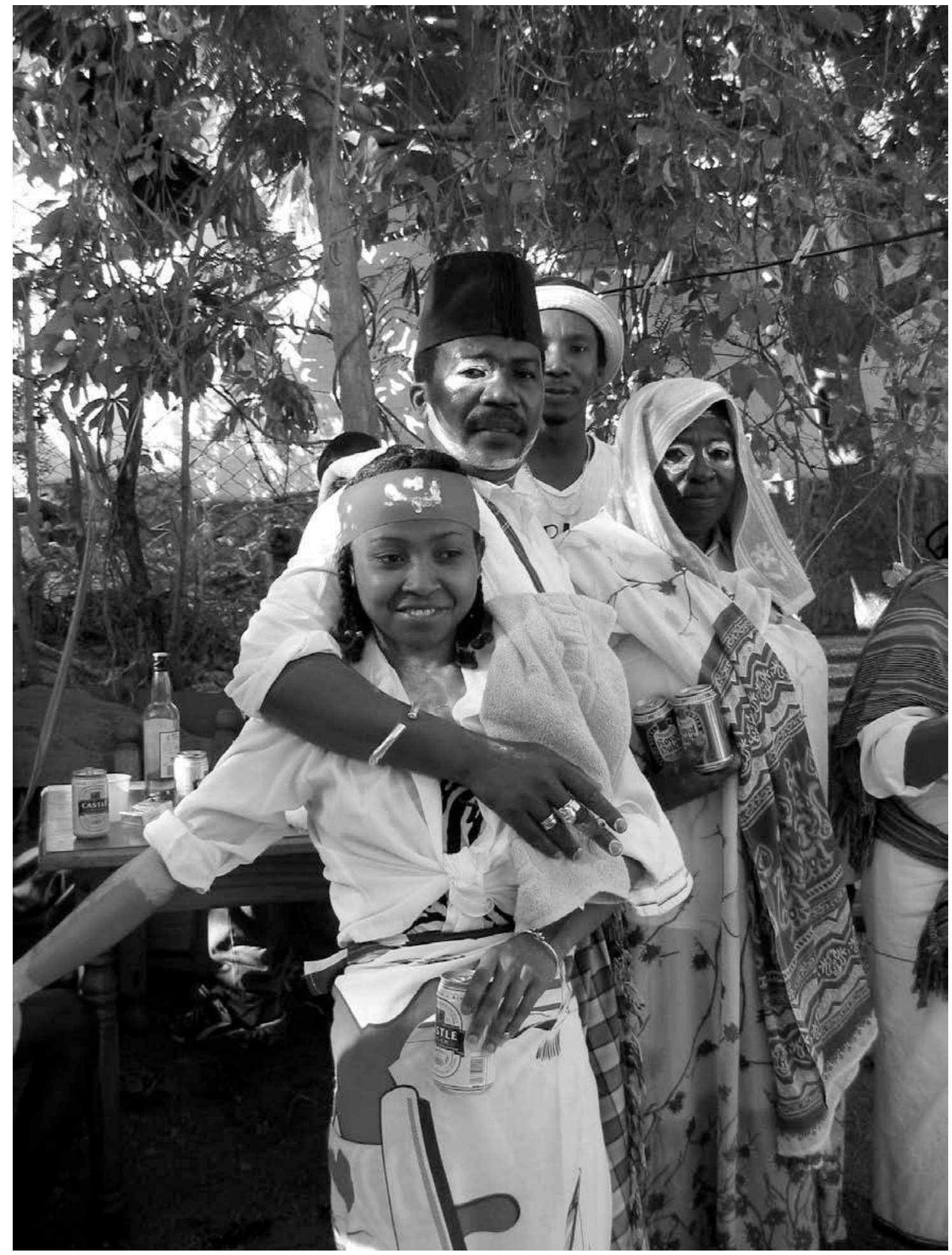

Fig. 1 Moment de détente au cours d'un rituel thérapeutique : les esprits tromba prennent bien volontiers la pose. Mayotte, juillet 2002. Photo Bertrand Hell (B. H.). 


\section{Négocier avec les esprits tromba à Mayotte Retour surle «théâtre vécu » de la possession}

Bertrand Hell

"Cette étude socio-psycho-biologique de la mystique doit être faite. Je pense qu'il y a nécessairement des moyens biologiques d'entrer en "communication avec le Dieu". " (Mauss 1950a [1903] : 386)

L a présente étude porte sur des travaux menés récemment à Mayotte mais s'inscrit dans une recherche amorcée dès 1981 au Maroc et consacrée au sens vécu de la possession. Un premier volet a consisté à analyser l'image d'alliés des esprits attachée aux officiants et à explorer «l'inquiétante étrangeté " dans laquelle baignent des rituels construits autour d'un acte de métacommunication (Hell 1999). Bien vite s'est posée la question de cette "opinion qui crée le magicien et les influences qu'il dégage " (Mauss 1950a [1903] : 32). Être en transe et donner les signes de la possession n'est pas tout! Au Maroc, comme au Brésil ou à Cuba, on reconnaît sans ambages que, nonobstant la présence d'esprits identiques chez des initiés, les pouvoirs thérapeutiques et divinatoires varient grandement selon les cas. Et chacun de nous renvoyer à l'idée d'une "force » inégale des entités incorporées et, partant, à la valeur fluctuante de l'initiation. Le second temps de cette recherche porte donc sur le processus initiatique lui-même et en particulier sur le mécanisme de l'installation des esprits chez les néophytes. Sur ce dernier point, le terrain entrepris depuis 2002 à Mayotte s'avère d'une très grande richesse car, dans les deux principaux cultes de possession de cette île de l'archipel des Comores (le ngoma d'origine bantoue et le rumbu des tromba d'inspiration malgache), les fundi wa madjini (littéralement « maîtres ès djinns ») attribuent tou- jours une importance cruciale aux rituels dits « médicaments " (dalao), qui consistent à domestiquer progressivement les esprits jusqu'à leur contrôle abouti ${ }^{1}$. Tout en renvoyant à une transformation en profondeur de la personne, ces rituels comportent une soigneuse dimension théâtrale, à l'exemple de la grande cérémonie qui voit l'initié déclamer publiquement le nom de ses esprits. L'authentique et l'artifice sont ici étroitement imbriqués. Ce constat ethnographique invite à se pencher à nouveau sur le dossier ouvert, dès 1958, par plusieurs ethnologues français, à savoir Roger Bastide, Michel Leiris et Alfred Métraux. Comment rendre compte de ce "vestiaire de personnalités ${ }^{2}$ " que la possession offre aux adeptes? Quoique centrale, la question du jeu de la possession semble depuis lors être restée quelque peu en jachère. Je me propose ici de la réactualiser en tirant parti des nouvelles données relatives aux états modifiés de conscience et à la plasticité cérébrale. Pour ce faire, je m'appuierai sur un matériau ethnographique concernant le culte des tromba à Mayotte, dont la diffusion résulte de la présence, attestée dès le premier recensement officiel de 1852, de fortes communautés

1. À ce jour, ce terrain mahorais a fait l'objet de sept séjours pour un total de dix mois. Les missions ont été réalisées avec le soutien du CNRS et du Centre d'études interdisciplinaires des faits religieux (CEIFR) de l'École des hautes études en sciences sociales.

2. L'expression de Michel Leiris est citée par Jean Jamin (1996 : 40). 
malgaches (Sakalava et Betsimisaraka principalement) sur le territoire de cette " collectivité départementale française ${ }^{3}$ ». L'attention se focalisera notamment sur les deux rituels terminaux qui vinrent conclure en 2005 le parcours initiatique d'une mère de famille mahoraise, Machamou, ayant hérité huit tromba (les esprits malgaches) de sa grand-mère. Il s'agissait pour elle, une fois énoncés solennellement les fady (les interdits) propres à chaque esprit, d'entreprendre d'âpres négociations avec ces esprits pour lever partiellement les interdits et se rendre ainsi la vie quotidienne un peu plus facile.

\section{Des esprits mécontents}

L'histoire personnelle de Machamou réfléchit parfaitement la biographie classique des adeptes des cultes de possession dont les rituels s'apparentent, selon la formulation de Michel Leiris à propos du zar éthiopien, à " une sorte d'initiation dont le point de départ aura été la maladie » (1992 : 68). Cette maladie élective se manifesta chez Machamou dès la plus jeune enfance par des évanouissements soudains, des phases de prostration et d'asthénie. Les traitements médicaux ordinaires s'avérant inefficaces, un devin fut consulté. Celui-ci imputa l'origine des attaques à la présence de nombreux esprits

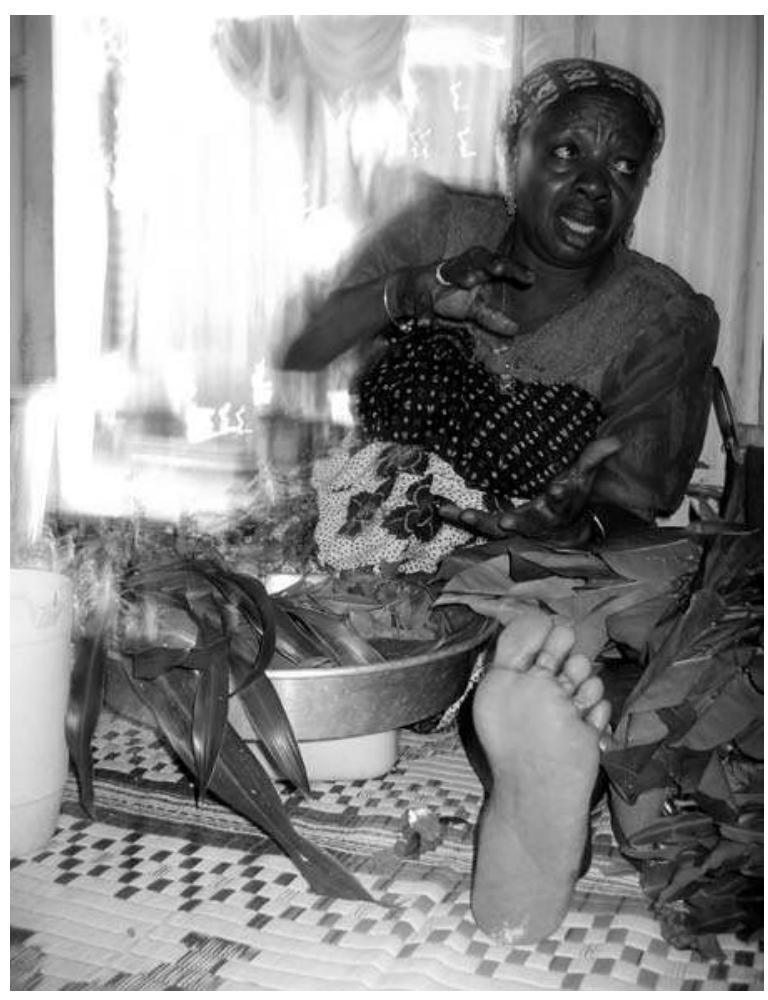

Fig. 2 Avant le rituel d'installation des esprits, les initiées préparent les plantes nécessaires aux différentes «bassines». Mayotte, août 2005. Photo B. H. chez ses ascendants : patros chez sa mère, tromba chez sa grand-mère maternelle. Différents « médicaments » permirent d'obtenir un répit jusqu'à l'âge de 20 ans, mais les troubles reprirent, menaçant sérieusement sa santé. Il fut alors décidé avec le fundi wa madjini de répondre en tout premier lieu aux exigences de ces patros impatients qui voulaient faire de la jeune femme leur «siège ». $\mathrm{Au}$ terme d'une cure initiatique de plusieurs années, Machamou put organiser en 1991 son grand ngoma, la nuit rituelle de possession, tenue sur une place du village, au cours de laquelle ses esprits patros déclamèrent leur identité. Depuis, Machamou officie elle-même comme fundi wa madjini, pratiquant la voyance et prenant en charge des patients. Mais la question des autres esprits familiaux, les tromba, restait en suspens.

De fait, à l'époque de notre première rencontre en 2002, Machamou pensait avoir trouvé un certain équilibre dans sa relation avec les tromba. Elle se contentait - le moins souvent possible - d'incorporer des changizy, ces esprits mineurs apparaissant sous la forme de marins morts noyés au xix siècle au large de Madagascar. Pour le reste, elle avait confié à Djalud, le plus puissant de ses esprits patros, le soin de la protéger de toute intrusion trop pressante des grands tromba ${ }^{4}$. Mais les choses ont pris une tournure plus chaotique ces dernières années. La possession par les changizy est devenue irrépressible et, surtout, s'exprime de plus en plus rudement : transformée en l'un de ces marins noceurs et paillards, Machamou boit de l'alcool outre mesure, titube, multiplie les grivoiseries et tient des propos obscènes. Malgré les conseils des autres initiés, elle refuse obstinément de se rendre à l'évidence et ne veut s'engager dans aucune alliance avec les tromba. La raison de ce refus farouche? « La honte ", me répète-t-elle à plusieurs reprises. Le commerce avec les tromba lui répugne en raison de leur penchant notoire pour les alcools forts et leur goût dénaturé de la fête. Whisky bu au goulot, musique criarde, danses lascives : tout ceci s'avère bien peu compatible avec le statut respectable de mère de famille (elle a eu cinq enfants) et de musulmane pieuse (elle a accompli le grand pèlerinage à La Mecque en 1999)

3. Tel est le statut officiel de Mayotte, achetée en 1841 par la France au sultan Andriantsoly. À l'heure actuelle, on évalue à $40 \%$ le pourcentage de Mahorais ayant comme langue première le shibushi, le dialecte malgache en usage localement (Maandhui 1996: 5).

4. Les tromba désignent originellement les ancêtres royaux de la dynastie sakalava ayant régné à Madagascar jusqu'au XIX ${ }^{e}$ siècle. D'autres esprits sont venus par la suite s'intégrer à ce culte de possession (les tromba antemoro, vezo, volafotsy, marins, etc.). Pour une étude de la relation entre le culte des tromba et la royauté sakalava, voir Michael Lambek (2002). 


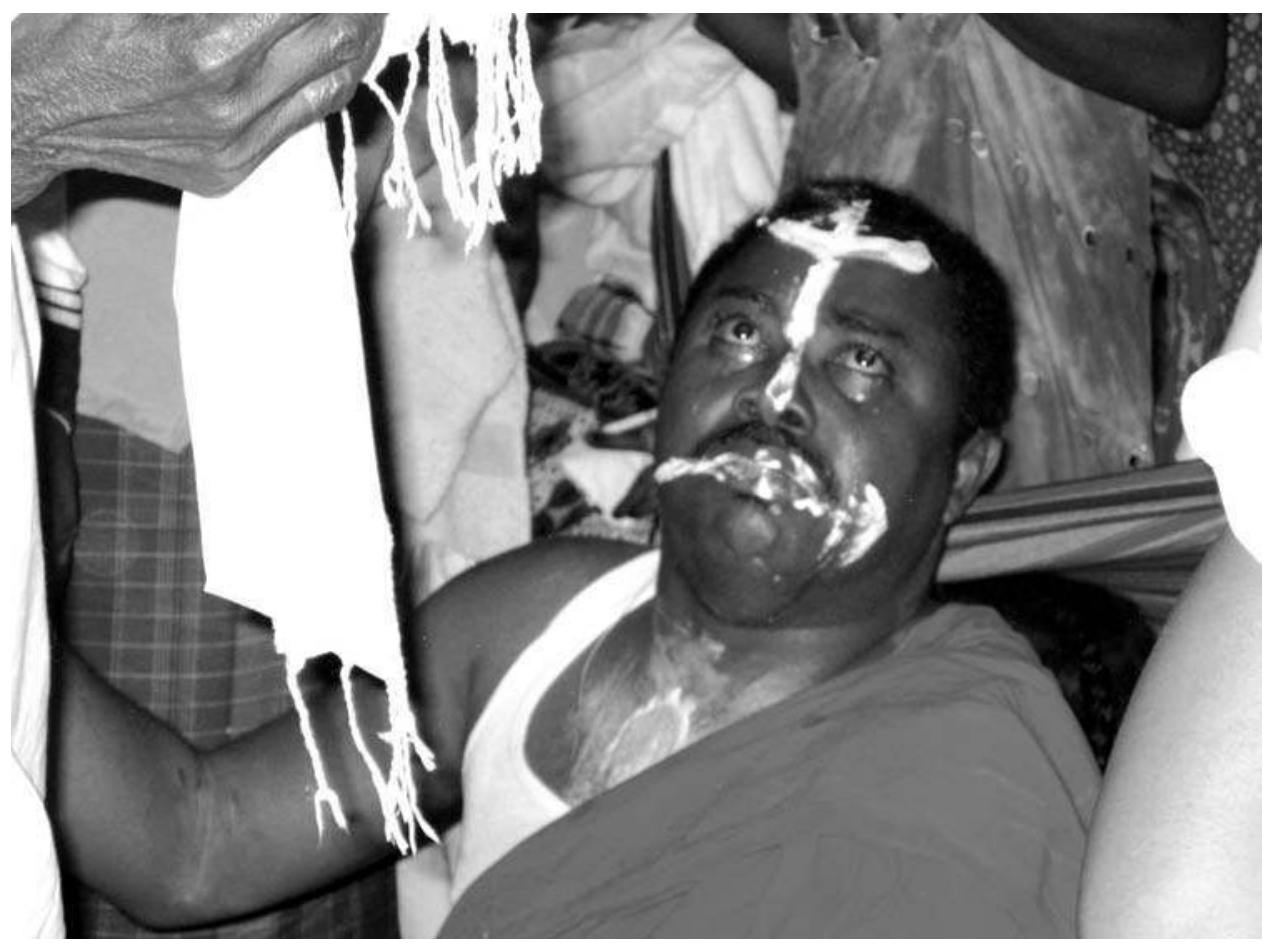

Fig. 3 Montée d'un esprit tromba sur un initié. Mayotte, novembre 2006. Photo B. H.

auquel elle aspire. La foule qui se presse lors des rumbu publics n'est-elle pas toujours prompte à dauber le comportement des possédés?

À présent, la menace des tromba se précise, l'état de santé de Machamou se détériore sérieusement. Elle se met à cracher du sang lors de violentes crises convulsives; chacun, dans le culte des adeptes, de reconnaître là l'action morbide de Ndranaverna, un prince tromba mort la poitrine fracassée. Se voiler la face plus longtemps serait lourd de conséquences. Machamou se résigne à l'évidence : les esprits n'ont que trop attendu, l'heure est venue de les installer.

\section{La retraite initiatique}

Nous sommes en août 2005. Après une série de rumbu-dalao étalée sur plusieurs mois, qui ont amené peu à peu les différentes entités à se manifester plus calmement et à formuler distinctement leurs exigences, il s'avère désormais possible de procéder au valihataka, le rituel d'autodésignation des tromba. La cérémonie est coûteuse (plus de six mille euros) et lourde à organiser car les esprits ont dressé une longue liste de leurs parents tromba à inviter ; ils requièrent la présence d'un orchestre célèbre et exigent des boissons et des cigarettes à profusion, y compris pour contenter la très nombreuse assistance attendue.
Ce grand rituel est obligatoirement précédé d'une période de réclusion totale de sept jours ponctuée de nombreux dalao dirigés par le fundi Attoumani. Cette semaine s'ouvre avec des ablutions purificatrices réalisées à l'aide de neuf seaux d'eau où baignent des herbes et des racines. Machamou doit vivre cloîtrée dans une pièce, seuls y sont admis les quelques initiés chargés de faire monter tous les jours ses tromba afin de les préparer à leur apparition publique. Application sur le corps de pâtes végétales, fumigations d'encens et onctions avec de l'eau lustrale mélangée à du kaolin, succession de « bassines » tantôt chaudes (sous forme d'inhalation de vapeur) pour forcer la venue d'un esprit indécis, tantôt froides (bains) pour, au contraire, modérer l'irruption et éviter les états de trop grande confusion : les rites s'enchaînent, sous le regard attentif du chef de culte, pour fortifier le corps de la novice et le rendre apte à incorporer les puissants tromba. Mais il s'agit aussi, grâce à des plantes "qui repoussent ", de faire refluer les esprits indésirables, ceux-là mêmes qui, toujours prompts à se glisser dans un corps fragilisé, viendraient lui souffler des propos ambigus. Et, jour et nuit, inlassablement, les rumbu (" battements de mains ») rythmés et les exhortations des initiés encouragent la venue des tromba. Machamou traverse cette période initiatique dans une sorte de léthargie éveillée proche de cet «état d'hébétude » attesté dans la plupart des cultes de 
possession ${ }^{5}$. Mais l'essentiel est survenu : les tromba se sont montrés sous leur vrai visage, ils ont donné leur assentiment pour la tenue du rituel public. Fundi Attoumani part prendre quelque repos, Machamou est prête.

\section{La présentation des esprits}

La cérémonie se déroule selon le scénario habituel des grands rumbu à finalité votive ou thérapeutique. Ici, pour accueillir la foule des invités, elle se tient juste devant la maison de Machamou, sur une large place de terre battue. À l'une des extrémités, soigneusement dressé vers le nord-est, se trouve l'autel des tromba avec les différentes offrandes (dont quatorze bouteilles de barisa, le breuvage sacré) et les objets cultuels (assiettes avec des pièces d'argent, pots de kaolin, cannes et sceptres, coupelles d'encens, etc.). Quelques chaises et des petits bancs de bois (" trônes" des rois tromba) sont disposés de part et d'autre, de grandes nattes sont étendues au sol. À l'autre extrémité, de jeunes musiciens peaufinent les derniers réglages devant une impressionnante sono : les tromba se plaisent dans le charivari le plus assourdissant. À Mayotte, les joueurs traditionnels de valiha (cithare tubulaire en bambou) et d'accordéon ont cédé la place aux orchestres modernes (batterie, guitares électriques, synthétiseurs) interprétant les derniers airs populaires à la mode. L'ordonnance du rituel ne paraît nullement avoir pâti de ce changement car, comme le remarque un observateur des cultes de Madagascar, ce qui importe avant tout c'est d'instaurer une ambiance festive : « Le bruit est de rigueur : un grand bruit dans certains cas mais toujours rythmé [...]. Le rythme de plus en plus accéléré a pour fonction de créer l'ambiance et de réveiller des esprits [...]. Ce n'est pas tant la parole, que le rythme et la musique qui semblent importer le plus [...] » (Jaovelo-Dzao 1997 : 338-340).

Le rituel débute vers $22 \mathrm{~h} 30$. Une foule bigarrée et bruyante, majoritairement des femmes, se presse sur les nattes. Après la série des litanies d'ouverture, les possessions se succèdent : d'abord les grands rois, puis les princes et enfin les roturiers. Les marins noceurs et autres changizi viveurs n'apparaissant, eux, que plus tard dans la nuit. Dès les premiers signes de la possession, on applique soigneusement de la pâte de kaolin sur les parties les plus douloureuses du corps de l'initié, puis on le dévêt afin de lui enfiler le costume de son tromba : pagne rouge, turban et lance pour ce roi guerrier, grosse chemise de toile, lamba (étole de soie) mal- gache traditionnel, canotier de brousse et bouteille de rhum pour ce riche propriétaire terrien ou encore robe de mousseline, voiles rose bonbon et jouet en bois pour ce tromba fillette. Chaque nouvelle apparition est saluée par des koesy ("salut ») sonores et les adeptes s'approchent pour présenter leurs hommages. L'atmosphère est avant tout à la fête. Les esprits exhortent l'assemblée à participer activement aux réjouissances : le kaolin utilisé aujourd'hui n'est-il pas appelé ravoravo, c'est-àdire " joie » ? Régulièrement, des vagues de battements de mains font vibrer la foule à l'unisson. Les tromba boivent, dansent et devisent librement avec les adeptes. Machamou, quant à elle, est assise devant l'autel au pied des rois et des reines hiératiques qui la cajolent, la tapotent dans le dos et l'aspergent de l'eau contenue dans les assiettes rituelles à chaque nouvelle transe qui la secoue.

Peu à peu, la «chaleur » apportée par les esprits gagne toute l'assistance. Il est 2 heures du matin et les possessions spontanées se propagent. Comme toujours, quelques femmes espiègles en profitent pour mimer une incorporation, déclenchant l'hilarité générale. Certaines n'hésitent pas à se lever et à imiter quelques gestes caractéristiques d'un esprit, sous le regard faussement courroucé des tromba. Quant aux hommes efféminés que tout cercle d'initiés inclut invariablement, il leur revient de multiplier les facéties, jouant le rôle de bouffons rituels. À présent, offertes par les esprits, les boissons alcoolisées circulent; les changizy se déchaînent, entraînant des femmes dans leurs danses effrénées. Étonnante atmosphère! D'un côté, la musique zouk, les rires et les épanchements dionysiaques nous plongent au cœur d'une fête apparemment profane, de l'autre, la déambulation dans la foule de ces étranges participants costumés, au visage grimé en blanc par le kaolin et aux gestes curieusement saccadés, nous rappelle qu'il s'agit bien d'un authentique culte de possession.

Il est 5 h 30, le jour point à l'horizon. L'instant crucial approche et Machamou s'est retirée dans la maison pour d'ultimes préparatifs. Encadrée par quelques initiés portant les habits neufs des tromba, la voilà qui réapparaît, le regard fiévreux, le pas chancelant, enveloppée dans un épais drap blanc. Le cortège fend lentement la foule et vient se placer devant l'autel au milieu des tromba. La musique s'interrompt, l'assemblée retient son souffle. La tension est palpable. Pendant l'heure et demie qui suit, les huit tromba vont successivement

5. Pour les cultes des orixas et vôdun, par exemple, voir Pierre Verger (1954: 171). 


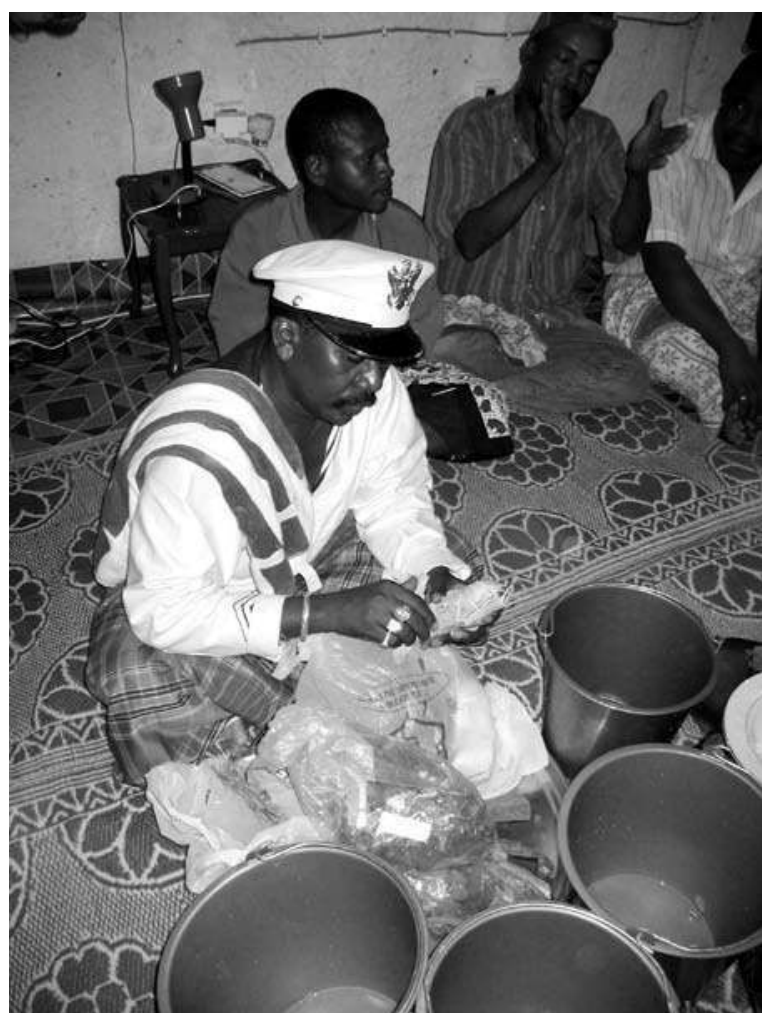

Fig. 4 Monté sur le fundi Attoumani, l'esprit tromba AlexandreLe-Marin prépare un "médicament " pour un rituel thérapeutique. Mayotte, décembre 2006. Photo B. H.

posséder leur « siège " qui, à chaque fois, revêt les vêtements adéquats. Puis il faut décliner avec clarté son identité et faire face aux interrogations pressantes des autres esprits qui font bloc autour d'elle. Des spectateurs curieux s'approchent discrètement pour mieux entendre le jeu des questions-réponses. "Comment s'appelle ton frère? »; «Où se situe ton doany [emplacement sacré »] ? " « Et moi, qui suis-je ? » « Ton fady du poulet: blanc ou rouge? " S'ils sont satisfaits, les tromba congratulent le nouvel arrivant, l'invitent à saluer l'assistance et à esquisser quelques gestes ou pas de danse caractéristiques de leur histoire personnelle $e^{6}$.̀̀ la sourde appréhension succède à présent une franche allégresse. Les proches de Machamou s'étreignent, certains sont en pleurs. On remercie les esprits avec effusion. La musique repart de plus belle et la fête reprend. Machamou incorpore le tromba Ndranaverna, qui se lance aussitôt dans une tournée de salutations avec le public. Le rituel ne prend fin que vers 10 heures du matin, une fois le partage des offrandes effectué entre les esprits (packs de boisson, cartouches de cigarettes, enveloppes nominatives contenant quelques dizaines d'euros). Machamou devra, elle, accomplir une courte réclusion ponctuée de quelques «bassines ».

\section{Du bon usage de la transe}

La fonction sociale de cette véritable cérémonie d'adoubement se dévoile clairement. Devant ce parterre comprenant aussi bien les principaux tromba connus à Mayotte que la foule des adeptes, Machamou exhibe publiquement sa connaissance des règles du jeu. En respectant l'ordre de préséance des apparitions de tromba, elle montre sa faculté de "se soumettre à un protocole assez strict ", tout comme la possédée du zar (Leiris 1989 [1958] : 121). Ses réponses précises aux questions rituelles prouvent que, à l'instar de la yao du candomblé brésilien, elle est passée « de l'expérience empirique au savoir organisé » et qu'elle s'est parfaitement « familiarisée aux devoirs et aux obligations de sa future charge " (Bastide 2000 [1958] : 65). Enfin, sa maîtrise des comportements ostentatoires propres à chaque esprit atteste cette aptitude dont doivent faire preuve tous les initiés, à l'instar de ces hounsi haïtiens dont « le talent se révèle en particulier lors des possessions successives qui les obligent à changer sans transition de rôle " (Métraux 1958 : 114). Assurément, l'initiation doit se lire sous cet angle comme une quête de légitimité dont l'observance de la théâtralisation des possessions constitue une étape obligatoire.

Ce dont témoigne Machamou au cours de ce rite de passage se situe aussi à un autre niveau. Nombreux sont les adeptes qui furent témoins, ces dernières années, des possessions désordonnées et «honteuses » qu'elle dut endurer lors de rumbu publics. Quant à ses récents ennuis de santé, ils ne sont un secret pour personne sur l'île. Aujourd'hui, la nouvelle initiée exhibe une personnalité insoupçonnée. Une force certaine est requise pour contrôler, en un si court laps de temps, ces différents états successifs de dépersonnalisation totale, et ce tout en flottant dans une profonde hébétude. D'ailleurs, il arrive que des novices ne parviennent pas à franchir cette étape cruciale alors même que toutes les conditions pratiques sont réunies pour réussir leur initiation (argent disponible pour le rituel, connaissance des rites, etc.). Si, dans la lignée des travaux d'anthropologie théâtrale de Jerzy Grotowski, Machamou peut être assimilée à une « performer » (Pradier 2005), cette performance est aussi jaugée à l'aune d'un processus psychique souterrain, en l'occurrence la réorganisation du Moi à partir d'expériences intimes hautement bouleversantes et déstructurantes. Cette idée du " travail avec les esprits "

6. Tel tromba boite car il s'est fracturé les jambes, tel autre, joueur d'accordéon professionnel, agite les bras de manière saccadée, tel autre encore, boxeur réputé, fait étalage de son jeu de jambes. 
n'est donc pas une simple figure de rhétorique. Employée de manière parfaitement identique dans le langage ordinaire, aussi bien à Mayotte que chez les Gnawa marocains, cette formulation réfléchit la réalité d'un work in progress qui ne peut se découvrir que dans l'intimité des petits rumbu-médicaments et des périodes rituelles de réclusion. Ainsi, grâce à la relation de confiance établie avec le fundi Attoumani, il m'a été possible de suivre, outre celle de Machamou, quatre autres retraites d'initiés. Une relation de confiance? Ici, l'observation participante a pu dépasser les limites qu'imposent habituellement les systèmes initiatiques. À vrai dire, ce fundi semblait avoir compris assez rapidement que le fait d'assister à des répétitions du rite de la dénomination des esprits, dans «le secret » des pièces interdites aux non-initiés, n'allait pas inéluctablement me conduire à en conclure à l'inauthenticité du culte, voire à l'imposture. L'important pour lui était de me laisser entrevoir la complexité de cette relation interpersonnelle devant se jouer entre le novice, le chef de culte et le cercle restreint des initiés. Bref, à moi de décrypter ici une expression ethnographique, à échelle réduite, du célèbre triangle de la magie évoqué par Claude Lévi-Strauss (1958 [1949] : 192).

«Il faut qu'on ait déjà l'idée d'être possédé pour être possédé ", remarquent Jean Jamin et Michel Leiris en évoquant la démystification qu'implique parfois l'ethnologie (Leiris 1992 : 48). L'intériorisation au plus profond de l'être de cette idée s'opère dans ce cadre très précis des rituels ordinaires du culte. Et «dans la durée", précisent les initiés. Le procédé psychologique s'accompagne parfois d'un véritable apprentissage de la gestuelle adéquate, comme l'illustre cette séance de « travail » tenue au domicile du fundi en décembre 2006. Un homme malade s'était présenté à lui et son état nécessitait d'urgence un rumbu thérapeutique. Profitant de cette occasion, Attoumani avait convié trois jeunes filles à se joindre au petit groupe de ses assistants. Âgées d'une vingtaine d'années, celles-ci venaient tout juste d'amorcer leur initiation et pour l'heure en étaient au stade des possessions sauvages douloureusement vécues. Durant la majeure partie du rituel, l'attention du fundi et des tromba "montés sur " les assistants se focalise sur le patient car il s'agit d'identifier l'esprit responsable de ses maux à partir de l'indice le plus ténu. Comme la dizaine d'adeptes présents dans la pièce, les trois jeunes filles multiplient les battements de mains pour accompagner la musique de la petite chaîne stéréo. Après trois heures d'effort, le fundi semble satisfait et donne les indications pour que le malade effectue une série de « bassines » dans la cour. Le rumbu se poursuit. Attoumani incorpore Alexandre, le tromba marin, qui ne va pas tarder à distribuer bières et cigarettes à la ronde. Plusieurs autres initiés sont possédés à leur tour et le rituel thérapeutique cède la place à une fête improvisée des tromba. Attirés par la musique et le bruit, les voisins se pressent sur le pas de la porte pour voir danser les esprits. Les battements de mains redoublent d'intensité, la "chaleur " monte et les trois néophytes ne tardent pas à montrer les signes de la possession. Les deux premières connaissent leurs crises habituelles : assises, elles se mettent à trembler violemment et à crier en agitant frénétiquement les bras en l'air. Aussitôt, on les asperge d'eau mêlée de kaolin, appelée ici fangala nintsy («qui enlève le froid»), ce qui a pour effet de les plonger dans une profonde prostration entrecoupée de sanglots. La troisième, en revanche, se met à pousser des petits cris étouffés, elle semble vouloir happer de l'air et tombe ensuite, de tout son long, inanimée au sol. Sur un signe d'Alexandre, on la recouvre entièrement d'un tissu rituel, ce geste marquant l'arrivée d'un grand tromba. Des convulsions font frémir le linceul, les jambes de la néophyte jaillissent, étrangement raidies. Sur-le-champ, Alexandre les badigeonne soigneusement de kaolin tout en invitant l'assistance à multiplier les battements de mains. La possédée se redresse et fait mine de se relever en poussant les mêmes cris. On s'écarte, mais Alexandre la repousse de la main et lui verse le contenu de l'assiette sacrée sur la tête, ce qui déclenche une crise spectaculaire de tremblements. Après une longue minute de transe, elle s'affaisse une seconde fois, inconsciente. Pose du tissu, onction des jambes : les gestes sont renouvelés mais, à présent, une fois redressée, la possédée tourne la tête de gauche à droite, hagarde et semblant implorer de l'aide. Un adepte s'approche et, lui saisissant les mains, l'aide à se tenir droite. On l'entoure, lui enfile une chemise blanche, lui passe un lamba autour des reins et Alexandre lui trace plusieurs signes sur le visage à l'aide du kaolin. Chacun en connaît la signification. Ces traits dénotent l'apparition de Ndramadanti, ce roi mort paralysé dont nul n'ignore qu'il ne peut se mouvoir seul. On l'aide donc à se rasseoir. Il restera présent, hiératique et complètement muet pendant une dizaine de minutes avant de se retirer de son « siège », laissant la jeune fille épuisée et sanglotante, affalée dans les bras d'une spectatrice. Tard dans la soirée, Attoumani me fera part de sa satisfaction. Pour cette néophyte, une étape importante a été franchie et son rituel d' " ouverture de la bouche » (vaky-yava) devient envisageable dans un avenir proche. 
La performance accomplie par Machamou lors de son rite de passage s'avère donc de la même veine que celle attendue des novices béninoises du culte des vôdun au moment de leur sortie de l'enclos où elles étaient recluses depuis plus d'un an. Devant le village rassemblé pour cet événement, elles doivent monter tour à tour sur des tabourets et " faire la démonstration de leur talent de danseuses " (Rouget 2006a :13). Sacré et théâtralité s'entremêlent dans les cultes de la possession, faisant naître des sentiments ambigus chez l'ethnologue. Ainsi Lydia Cabrera évoque une « sainte mystification " à propos des possessions par les orishas à Cuba (2003 [1954] : 51). Comment penser cet oxymoron?

\section{À propos du jeu liturgique de la possession}

La question de l'authenticité n'a pas échappé aux premiers ethnographes de métier confrontés à des rituels chamaniques. Ainsi, dès 1916, Bronislav Malinowski s'interroge sur la transe d'un médium de l'archipel des Trobriand : «Est-il un artiste ou un prophète ? " 1933 [1916] : 180), tandis que, peu après, à propos d'un chamane yakoute, Gavriil Ksenofontov salue le « véritable art théâtral qui tend à donner vie aux esprits » 1998 [1928] : 27). Mais c'est en France, en 1958, que s'ébauche une véritable réflexion collective autour du thème possession et théâtralisation. À cette date très précise, paraissent trois ouvrages classiques dédiés à différents cultes des esprits : Le Vaudou haïtien d'Alfred Métraux, Le Candomblé de Bahia de Roger Bastide et La Possession et ses aspects théâtraux [...] de Michel Leiris.

Ces trois études abondent en remarques relatives à la « volonté intérieure » des possédés, à la « censure exercée » ou encore au « jeu bien réglé » du scénario imposé par la tradition mythique. Selon un véritable effet de miroir, certaines expressions viennent parfaitement se croiser : pour Bastide la danse des orixas est « un opéra fabuleux » (2000 [1958] : 220), Métraux s'attache à l' « élément de comédie » que comporte le rituel tandis que Leiris y souligne la part singulièrement étendue « de l'art et du jeu » (1989 [1958] : 129). Toutefois, originalité marquante, les auteurs ne se bornent pas à gloser sur l'artificialité du phénomène. Ainsi, dans le même chapitre, Métraux prend soin de faire se répondre d'un côté des notations sur la présence dans les sanctuaires de " coulisses de théâtre " propres aux exigences vestimentaires du vaudou, et de l'autre une anecdote illustrant la «force de conviction » se manifestant chez les possédés eux-mêmes. Toute à sa possession par la déesse Ezili, une jeune femme avait généreusement distribué ses

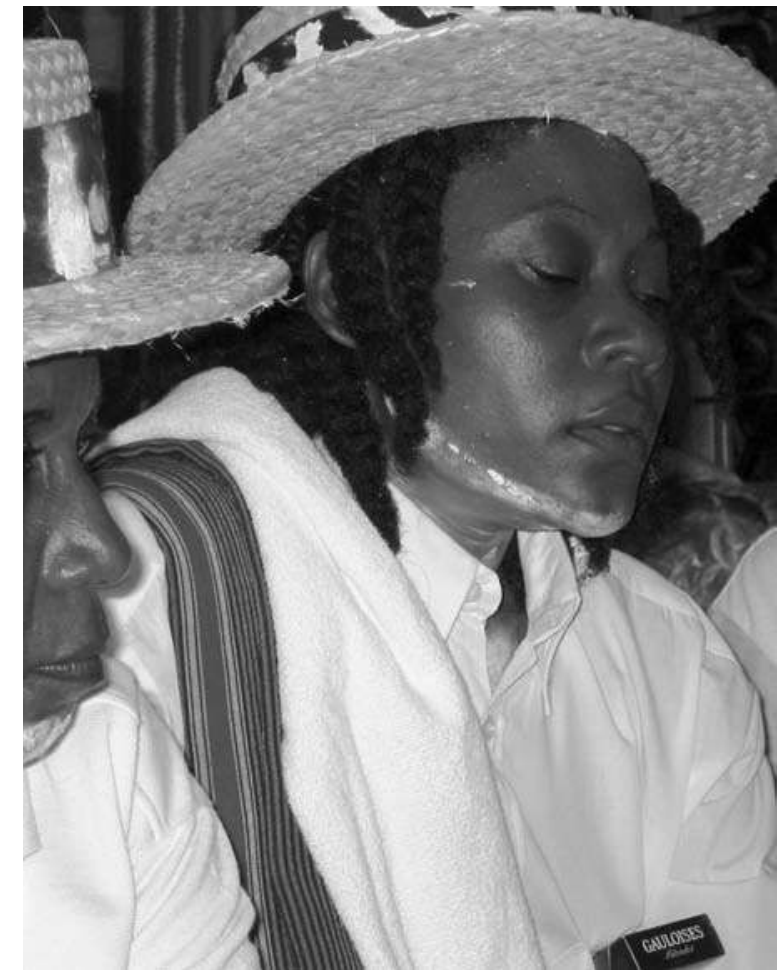

Fig. 5 Monté sur Machamou l'esprit du prince Ndranaverna écoute l'avis des autres tromba discutant de la levée des interdits, Mayotte, décembre 2006. Photo B. H.

économies à un partenaire de danse anonyme choisi dans l'assemblée. Le lendemain, regrettant amèrement cet argent gaspillé, elle assigna le jeune homme en justice. Mais bien que le juge ait tranché en sa faveur, elle refusa cependant de reprendre son offrande, réalisant qu'elle risquait de s'attirer les foudres de la déesse. Voilà pourquoi, estime l'ethnologue, les transes rituelles posent de fait « un problème fondamental ». Certes, le possédé, manifestement, « s'improvise acteur »; force est cependant de reconnaître " qu'il entre dans la peau de son personnage [et qu'il] tient son rôle de bonne foi " (Métraux 1958 : 106-125). La question n'est donc pas de tracer la frontière entre «le subi et le simulé » dans la transe, mais de se pencher sur le champ de gravitation au sein duquel se définit cette «bonne foi » du possédé. Le point de vue de Métraux n'est pas étranger à la présence de Leiris à ses côtés pour une mission ethnographique menée en commun en Haïti en 1948. Dans son carnet, ce dernier évoque un déjeuner « où je parle toujours vaudou : comme l'esprit possesseur intervient à la fois comme explication de l'état et comme formulation ou stylisation de cet état; rapport de la possession - être autre chose que ce que l'on est - avec le bovarysme et avec la mauvaise foi sartrienne [...] » (Jamin 1996 : 43). Bastide, pour sa part, retient le terme de « délusion », renvoyant 
au concept introduit par Pierre Janet dans la littérature médicale et qui interdit, selon lui, de réduire la transe religieuse à une simple simulation (2000 [1958] : 222).

\section{Un débat avorté}

Cette interrogation novatrice ne va pas se prolonger. Alfred Métraux disparaît en 1963, Michel Leiris s'engage dans l'écriture et n'entreprend plus aucun de ces voyages ethnographiques qui, nous rappelle Jean Jamin, " techniquement, voire logiquement auraient pu s'imposer à lui » (1996: 55). Seul Roger Bastide poursuit la réflexion sur le «jeu liturgique », insistant en particulier sur les idées du " contrôle de la transe ", c'est-à-dire sur «l'ensemble des séquences qui vont conditionner ce futur jeu de rôles » $(1975: 213)$. Mais cette perspective n'est désormais plus centrale, elle concourt essentiellement à étayer la distinction proposée entre sacré sauvage et sacré domestique.

En France, la problématique du jeu de la possession entre authenticité et facticité semble épuisée, aucune nouvelle étude de terrain ne venant alimenter la discussion. Pourquoi un tel désintérêt après 1958 ? Dans un article consacré à la réception en France des œuvres d'Ernesto De Martino - « un rendez-vous manqué »-, Daniel Fabre nous fournit des éléments de réponse. Car il y a effectivement tout lieu de s'interroger aussi sur l'absence de débat de fond qui suit la traduction, entre 1963 et 1971, des remarquables travaux sur la magie et le culte de possession des tarentulés en Italie du Sud proposés par le grand ethnologue italien. Ce silence n'est en fait pas étonnant. Le retour en France en 1947 de Claude Lévi-Strauss entraîne une profonde transformation intellectuelle " au point qu'en 1960 tout le paysage de l'ethnologie française était remodelé sans que les acteurs en aient une conscience nette et complète » (Fabre 1999 : 230). Le structuralisme qui prédomine ouvre d'autres débats, en particulier avec le marxisme et l'herméneutique, dans lesquels ni la production demartinienne ni celle de " cette frange originale de l'ethnologie française que l'on a parfois qualifiée de "poétique" » ne trouvent désormais de place (ibid. : 210).

À vrai dire, Lévi-Strauss s'est dans un premier temps intéressé à la question. Même si aucun des trois ouvrages de 1958 cités précédemment n'en porte mention, il a, dès 1949, dans Le Sorcier et sa magie et dans L'Efficacité symbolique, posé de solides jalons dans la compréhension du " complexe chamanistique ». S'interrogeant par exemple sur le spectacle offert à l'auditoire, il remarque : « Mais le mot de spectacle ne doit pas tromper : le chamane ne se contente pas de reproduire ou de mimer certains événements; il les revit effectivement dans toute leur vivacité, leur originalité et leur violence " (1958 [1949] : 207). Le chantier esquissé est resté en l'état pour deux raisons. D'une part, le parallèle établi entre cure chamanique et cure psychanalytique, qui pose le chamane comme " un abréacteur professionnel ", ne trouve aucun prolongement suite à un sentiment croissant de défiance vis-à-vis de la psychanalyse ${ }^{7}$. Explorer le fossé qui se creuse entre le structuralisme et, entre autres, la pensée lacanienne, nous entraînerait hors de notre propos. En revanche, la seconde raison nous intéresse très directement car elle s'avère lourde de conséquences quant à la conception même du travail de terrain. Le paradigme structuraliste n'accorde aucune importance à la recherche du sens donné par les acteurs aux constructions symboliques car, comme l'énonce Lévi-Strauss à propos de l'analyse des mythes, celle-ci "n'a pas et ne peut avoir pour objet de montrer comment pensent des hommes » $(1964: 20)$. Construire un objet anthropologique grâce à l'observation minutieuse des attitudes des possédés et à partir d'un suivi patient de leur parcours de vie, voilà qui ne présente désormais guère d'intérêt heuristique. Bien plus, la légitimité même d'une telle observation participante est contestée. Cette conception de faire science renvoie explicitement à la "grandeur " des sciences dures ${ }^{8}$. Elle impose au chercheur une distance, une extériorité qui rend suspectes, pour l'étude du phénomène de la possession, l'approche " poétique » des ethnologues français et la démarche d'insider de Zora Neale Hurston (pour le voodoo de La Nouvelle-Orléans, 1935) ou celle empreinte de « cordialité méthodologique ${ }^{9}$ " de Lydia Cabrera (pour les religions afro-cubaines, 1954).

7. Très vite, la technique thérapeutique comme la construction théorique cessent de séduire Lévi-Strauss : « Surtout j'ai voulu m'opposer à la tentation qu'éprouvent trop d'ethnologues, de sociologues ou d'historiens qui, quand leurs interprétations tournent court, trouvent commode, au lieu de les remettre en chantier, de combler les vides devant lesquels ils se trouvent avec ces explications passe-partout dont la psychanalyse est prodigue » (Eribon $1988: 151$ ).

8. « C'est pourquoi la réflexion scientifique, telle qu'elle se manifeste dans toute sa grandeur - dans la biologie ou dans la physique - me sert de phare » (entretien de Claude Lévi-Srauss avec DominiqueAntoine Grisoni, Magazine Littéraire, 2003 : 17). Sur la rupture qu'opère cette nouvelle conception de la scientificité dans le champ de l'ethnologie française, voir aussi Vincent Debaene (2006).

9. L'expression est d'Erwan Dianteill dans sa préface de la traduction française du livre de Lydia Cabrera (2003 [1954] : 11]. On trouvera également chez ce même auteur une discussion intéressante sur la question «du bon usage sociologique de la participation religieuse » dans son étude des cultes afro-cubains (Dianteill 2000 : 23-28). 


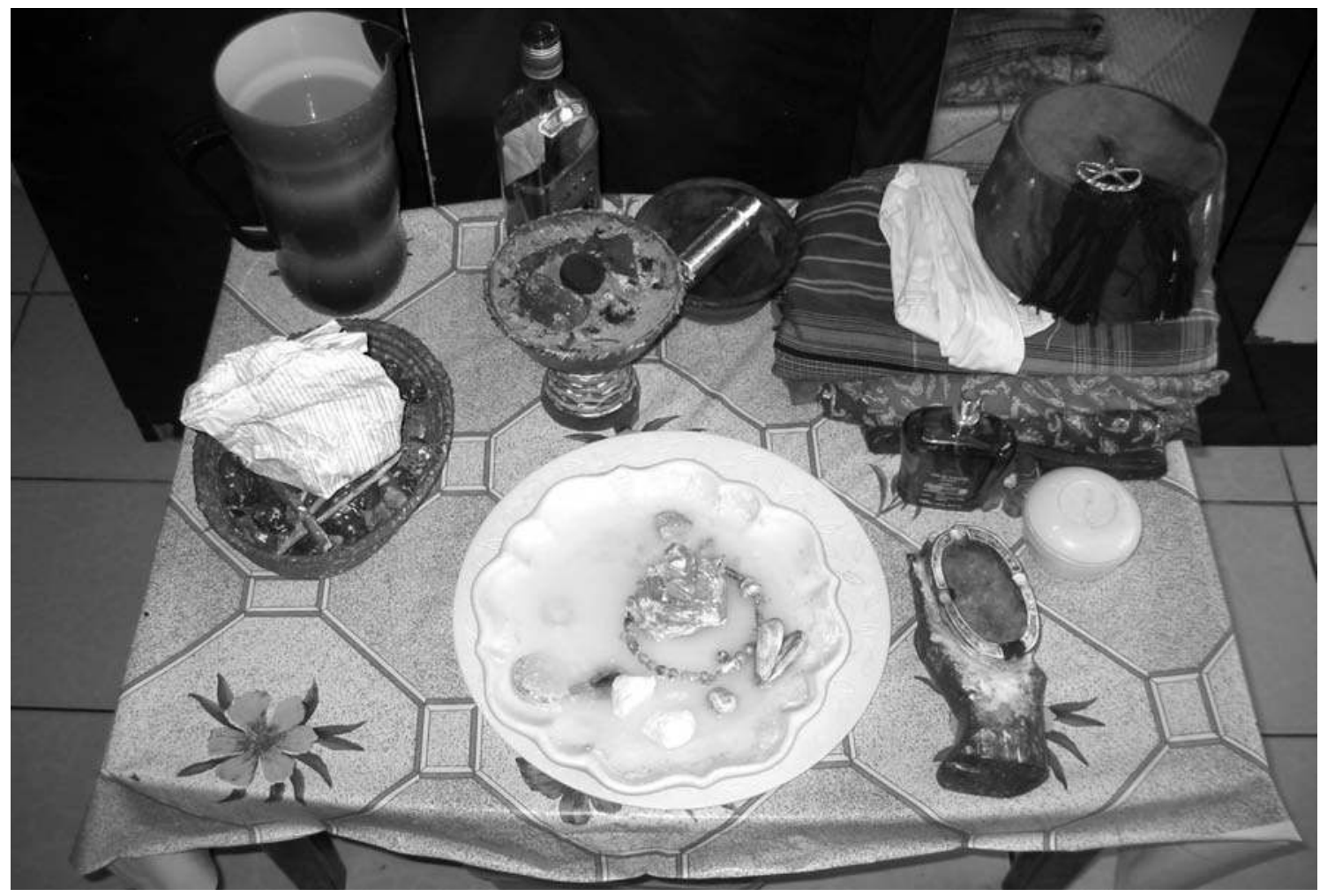

Fig. 6 L'autel du tromba Ndrankenraza avec son assiette divinatoire et ses vêtements rituels. Mayotte, décembre 2006. Photo B. H.

Ce profond remodelage de la recherche anthropologique en France conduit désormais les études sur la possession à se définir selon deux perspectives principales qui, à nouveau, réifient l'opposition authentique-inauthentique. Le débat sur la transe entre Gilbert Rouget et Roberte Hamayon cristallise parfaitement ce clivage. Soit la transe correspond à « une disposition psychophysiologique innée de la nature humaine » (Rouget 1990 [1980]: 39), soit elle ne renvoie à aucun « état » ni à aucune « expérience » vécue, mais procède d'un jeu de rôles (Hamayon 1995a : 420). La transe devient l'enjeu intellectuel de la discussion entre le structuralisme et le fonctionnalisme ${ }^{10}$. Pour les structuralistes, elle est un fait de nature universel sur lequel se sont élaborés les deux scenarii du chamanisme et de la possession, dont la position inverse et symétrique répond à la loi binaire ordonnant les productions symboliques de l'homme. En conséquence, les possédés sont nécessairement passifs, ils sont livrés à une hébétude qu'ils ne maîtrisent en aucune façon. La transe, insiste Luc de Heusch, ne peut être qu'induite, « somnambulique " et donc structurellement différente de celle des chamanes, « auto-induite et hallucinatoire » (2006: 88). Du côté des fonctionnalistes, on privilégie les idées de mise en scène, de re-présentation, d'action rituelle permettant soit de légitimer une institution de métacom- munication, soit d'offrir un espace de régulation des tensions sociales. Ainsi, pour les cultes de l'océan Indien, Michael Lambek s'attache prioritairement au processus de gaining a voice permettant aux femmes mahoraises - majoritaires dans les cultes de possession - d'asseoir leur rôle social : « Les femmes jouent un rôle actif dans la possession [...] car cela leur donne plus d'autorité et de capacité d'action dans des activités auxquelles elles se sont toujours intéressées ${ }^{11}[. .$.$] " (1993:334). Gérard Althabe$ insiste pour sa part sur la dimension politique du phénomène à Madagascar, en soulignant la fonction de contestation et de libération dans l'imaginaire dévolue à ces cultes dans des sociétés souhaitant s'affranchir du double poids de la christianisation et de la colonisation (1969). Jean Poirier fait plutôt ressortir cette «sorte de thérapeutique collective " qui fait du possédé « un porteparole du groupe qui assure inconsciemment une fonction de régulation ou de censure sociales » $(1987: 287)$.

10. Dans la dernière pièce qu'il verse au dossier, Gilbert Rouget mobilise de manière convaincante les récents travaux des neurosciences relatifs à l'émotion, rompant avec une conception purement psychopathologique de la transe (2006b).

11. «Women are active in possession [...] because it gives them greater scope and authority in activities in which they have always taken an interest $[. .$.$] . »$ 
Toutes ces contributions présentent un intérêt évident. Nonobstant, leur focalisation sur une problématique particulière contribua à entraver le développement de nouveaux objets en ethnologie religieuse. Pour sortir de cette ornière, certains chercheurs choisirent d'autres chemins. Tel est le cas d'Élisabeth Claverie dans sa minutieuse analyse du pèlerinage marial de Medjugorje (2003). N'est-il pas significatif que cette « anthropologie des apparitions ", étayée par un véritable terrain ethnographique entrepris en 1987 , se soit progressivement construite à partir de concepts situés en dehors du champ classique de l'ethnologie française : le positivisme logique, la pragmatique du langage, la sociologie de la critique, et ce au détriment, par exemple, de l'apport de De Martino sur l'efficacité du symbolisme mythicorituel comme technique de reconfiguration de l'univers quotidien?

En ce qui concerne les transes, ce «rendez-vous manqué " concerne également l'anthropologie de la conscience. Contrairement aux États-Unis où cette perspective se révèle particulièrement dynamique et pleinement intégrée dans le champ officiel de l'American Anthropological Association (AAA), il n'existe en France aucun travail comparable à celui de Michael Winkelman sur les aspects neurophénoménologiques du chamanisme (2000) ou à celui de Judith Becker sur le rôle de la musique dans l'émergence de la trance persona (2004). Dans cette dernière étude, la spécialiste des cultes de possession de l'Asie du Sud-Est propose aux anthropologues d'intégrer les plus récentes découvertes sur le cerveau des émotions : « Puisque l'émotion joue un rôle central dans le concept de "conscience profonde" de Damasio, puisque les émotions organisent un ensemble complet d'activités chimiques et nerveuses qui affectent le corps entier, les émotions ne pourraient-elles pas jouer un rôle clé dans la perception d'une personnalité seconde dans notre propre corps? L'émotion liée à l'écoute musicale ne pourrait-elle pas également jouer un rôle central dans ce processus magique et mystérieux ${ }^{12}$ ? » (ibid. : 149). Les travaux de neurophysiologie invitent donc à prendre en compte l'élargissement considérable du champ d'étude des potentialités naturelles du cerveau humain, et à remettre en cause l'opposition entre un état normal de la conscience et des états « altérés».

Cette idée avancée par l'ethnomusicologue américaine d'un processus magique et mystérieux à l'œuvre dans la transe ne déparerait pas dans nos ouvrages de 1958. Il me semble désormais possible de revenir sur l'ouverture proposée en son temps par l'« ethnologie poétique " française et en particulier sur le principe d'une possession pouvant se définir " comme un théâtre vécu, et non comme un théâtre joué ou comme l'expression d'un délire collectif " (Leiris 1989 [1958] : 124). Comment penser aujourd'hui ce concept de théâtre vécu ? Pour tenter de répondre, intéressons-nous à présent au second rituel terminal accompli par Machamou.

\section{Une négociation délicate}

5 décembre 2006. Il est 15 h 30 et voilà la trentaine de personnes réunies dans la maison de Machamou pour ce rumbu de rachat des fady. Chamou, sa fille, tourne, inquiète. Elle triture nerveusement la feuille de papier sur laquelle, hier, la famille a récapitulé la liste des tabous : le poulet, le lait, les œufs, le gingembre, des fruits, le contact avec des bébés, manger des restes, etc. Tout ceci est contraignant, d'autant plus que ces interdits viennent se surajouter à ceux édictés par les patros, ces autres esprits installés sur Machamou. Dans un coin de la pièce s'entassent les packs de cigarettes et de boisson destinés aux tromba. Les dernières économies ont été englouties dans ce rituel; Chamou et son mari eux-mêmes, jeune couple très récemment établi, y ont consacré leur épargne. L'enjeu est d'importance car une vie normale est quasiment impossible en respectant tous ces fady.

La cérémonie débute par la répétition des battements de mains destinés à faire monter les esprits. Aujourd'hui, outre Ndrankendraza venant sur le fundi Attoumani, quatre vénérables rois sont attendus, tous apparentés. $\mathrm{Au}$ bout d'une heure d'efforts soutenus de l'assistance, ils apparaissent et s'installent sur leur trône placé devant l'autel. Le conseil des sages est prêt, Machamou peut désormais incorporer successivement ses huit tromba afin que chacun d'eux exprime son sentiment. À chaque apparition, une discussion s'instaure. Après avoir présenté l'assiette rituelle comprenant les offrandes (quelques pièces de monnaie), Chamou énumère la liste des interdits et sollicite la compréhension du tromba. Les rois interviennent et appuient la demande. Mais les trois premiers esprits - deux princes et une vieille reine - se montrent intraitables. Du bout des lèvres, ils ont accepté de lever quelques interdits mineurs telle la consommation des restes de nourriture du repas de la veille (à condition que les aliments restent toutefois dans la marmite)

12. «As emotion plays a central role in Damasio's core consciounsness, as emotions organize a whole host of chemical and neural activities that affect the entire body, might not emotions also be a key to the experience of another persona within one's own body? And might the emotional musical listening play a central role in this magical, mysterious process? » 
ou celle d'un poisson de petite taille. Ndranaverna refuse, par exemple, de revenir sur le tabou du fruit de l'anacardier et n'accepte qu'un contact visuel accidentel :l'initiée peut, à la rigueur, le voir comme motif figurant sur un voile vestimentaire. Il se fait déjà tard, Chamou est désespérée, et dans la pièce surchauffée la tension est palpable. Les heures passées à négocier n'ont

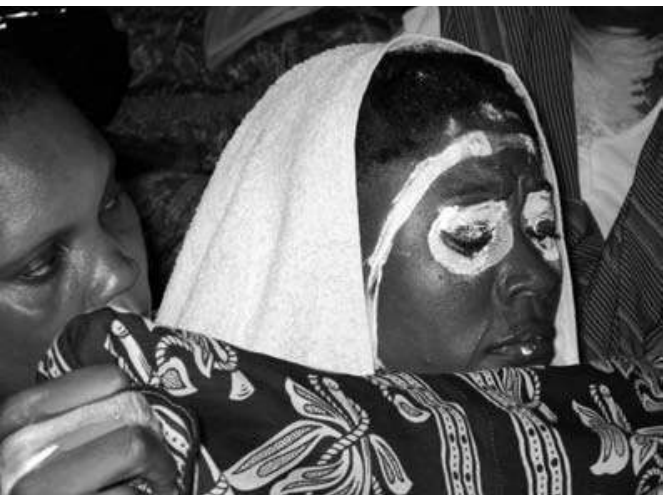

Fig. 7 Venue de Mona, un tromba féminin de la famille de Ndrankenraza. Mayotte, décembre 2006. Photo B. $\mathrm{H}$.

sa main s'est mise à enfler dangereusement et à se raidir peu après qu'elle a "emprunté " de l'argent dans l'assiette des esprits pour régler quelques courses urgentes. Plus grave, elle avait récemment terminé un plat dans la cuisine, par gourmandise, manquant ainsi à cette stricte règle de pureté alimentaire que lui impose le vieux patros Afrit. Elle se mit sur-le-champ à étouffer, déHeureusement, le tromba enfant Mona, la petite-fille de Ndrankendraza, qui possède à présent Machamou, semble mieux disposé à écouter le conseil des sages et surtout son grand-père, qui plaide la cause de la nouvelle initiée. Les choses s'arrangent. Mona accepte de lever les interdits les plus astreignants : le poulet (mais seulement de couleur blanche), le lait et les œufs, la patate douce (mais pas la « rouge »), les petites bananes à cuire (mais pas les sucrées). Enfin, elle se montre indulgente en ce qui concerne la « souillure » liée aux petits enfants. Machamou pourra s'occuper de temps en temps d'un nourrisson, à condition de prendre certaines précautions rituelles. Chamou retrouve le sourire : elle travaille toute la journée et le problème de la garde de son bébé est enfin résolu. Les incorporations reprennent. Mais ni le prince Leva, ni le capitaine de vaisseau Changuiz ne se montrent disposés à poursuivre la levée d'autres fady. "On lui a déjà trop accordé! » tonne ce dernier tromba à l'adresse de Chamou qui, penaude, plie sa liste en quatre. Quant à Alexandre puis à Chianti, les deux derniers esprits marins à posséder Machamou, ils n'auront d'autres préoccupations, à peine arrivés, que de boire de la bière, de badiner avec les spectateurs et de danser, tout en se plaisant à déclencher çà et là des possessions spontanées. Et tandis que la fête bat son plein au milieu des rires, des mimiques lascives et d'une musique poussée à fond devant l'autel, les membres de la famille vont défiler au pied des vieux tromba pour recueillir très respectueusement leurs bénédictions et écouter leurs recommandations. Ce n'est que vers 3 heures du matin, une fois le rituel achevé et son dernier tromba parti, que Machamou, totalement épuisée, prendra connaissance, avec une inquiétude manifeste, des résultats de la négociation.

Cette inquiétude peut se comprendre. En tant qu'initiée du culte ngoma, elle a déjà pu expérimenter dans la douleur les conséquences physiologiques de la transgression des interdits. Ainsi, il y a une dizaine d'années,

clenchant la panique dans la maison. Heureusement, quelqu'un eut le réflexe de téléphoner à son fundi, qui put prescrire un bain-médicament efficace.

\section{Nouveaux regards sur la conscience et l'hypnose}

Classiquement, la question des interdits conduit à pénétrer des cosmologies particulières. Elle peut aussi, comme le propose Rita Astuti pour les Vezo malgaches, nous éclairer sur la distinction morale-conventions (2007). À mon sens, les tabous liés aux esprits sont aussi susceptibles de mettre au jour certains ressorts du théâtre vécu de la possession. Il m'a été donné de suivre plusieurs « médicaments » effectués en urgence pour soigner des intoxications sévères, des paralysies ou des œdèmes faisant suite à une transgression. L'important ici est de relever que ce vécu corporel affecte essentiellement des initiés dont le statut d'allié des esprits impose des contraintes plus strictes. Sur ce point très précis, le terrain ethnographique rend problématique l'idée que « toute considération d'ordre physiologique et psychologique est inutile pour rendre compte du chamane " (Hamayon 1995b : 175).

Sans poursuivre plus avant, plusieurs auteurs ont évoqué le «conditionnement » (Bastide 1975 : 213) et l'« état de docile suggestibilité » (Verger $1982: 43$ ) du novice. Soucieux de creuser cette question, j'ai entrepris depuis 2002 une collaboration avec des spécialistes de l'hypnose m'ayant permis d'entreprendre une ethnographie de cures d'hynoanalyse ${ }^{13}$ et de participer à leurs travaux (Hell 2006). En tout premier lieu, ceci

13. Je remercie en particulier les psychiatres Édouard Collot du Groupement pour l'étude des applications médicales de l'hypnose (Paris) et Éric Bonvin de l'Institut romand d'hypnose suisse, le psychologue Jean-Roch Laurence de l'université Concordia (Montréal) et l'anthropologue Marlène D. de Rios de l'université de Californie (Irvine) pour leur aide précieuse. 
m'a permis de mesurer le fossé séparant les stéréotypes et les prénotions propres à l'essayisme de cabinet prévalant dans le débat psychanalytique français de la fécondité des études menées par les cliniciens et les chercheurs en neurosciences. Le développement récent des techniques de neuro-imagerie interdit, par exemple, d'entretenir plus longtemps la confusion entre «conscient » et « éveillé » ou « mentalement réceptif ». Les études sur l'hypnose viennent répondre à celles menées sur les états dissociatifs liés à la douleur (grands brûlés par exemple), sur la régulation des émotions qu'opère le système nerveux central ou encore sur les illusions perceptives. Sous cet angle, il est possible d'objectiver l'hypnose au niveau de l'activité cérébrale et de rompre ainsi avec l'idée d'un état de passivité lié à une seule force de suggestion. Très précisément, au niveau de l'induction hypnotique, on constate que « la détente mentale, le lâcher prise, conséquence de la réduction de la vigilance et de l'éveil, se traduisent par des modifications dans l'aire cingulaire antérieure, le tronc cérébral et le thalamus " (Collot 2006 : 114). L'hypnose renvoie donc à des états particuliers de rapport au monde et de perception dont la variabilité est illustrée par ses applications médicales. Sous une forme lourde, on peut faire appel à son pouvoir analgésique et développer les techniques dites d'hypno-sédation qui deviennent, dans certains hôpitaux, de véritables alternatives à l'anesthésie classique (CHUchotis 2006). Sous une forme plus légère, les transes silencieuses servent soit à travailler la " perceptude » du patient, soit à construire une alliance thérapeutique avec le soignant fondée sur l'intersubjectivité ${ }^{14}$. Le dénominateur commun de tous ces états hypnoïdes réside dans une modification du champ attentionnel et une concentration sur certaines représentations mentales propres. Or il apparaît que cette focalisation cérébrale peut induire très directement des réactions physiologiques tangibles. Voilà le point qui nous intéresse.

\section{État hypnoïde et revécu corporel}

Grâce aux récentes études par tomographie à émission de positrons menées simultanément dans plusieurs centres de recherche (cyclotron à Liège, universités d'Harvard et de Waterloo au Canada), l'existence de corrélats neuronaux spécifiques à l'hypnose a été démontrée ${ }^{15}$; il est ainsi possible désormais de tracer une frontière précise entre le souvenir et le revécu. Lorsqu'un sujet éveillé se remémore un instant de sa vie, il active surtout les lobes temporaux droit et gauche, aires qui ne réagissent pas lorsqu'il ne pense à rien de précis. En revanche, en état hypnoïde, le sujet mobilise un réseau cérébral comportant les régions de la vision (occipitale), des sensations (pariétale) et de la motricité (précentrale). Alors même qu'il est immobile, il voit, sent et bouge. Les données scientifiques viennent ainsi corroborer le rapport subjectif des hypnotisés, qui mentionnent invariablement l'impression de "revivre " les moments choisis, et non simplement de «s'en souvenir » (Faymonville et al. 2005 : 47). Des résultats identiques apparaissent lorsqu'il est proposé au sujet de se rappeler un morceau de musique ou une couleur : l'illusion sensorielle laisse des traces cérébrales précises montrant que l'état hypnoïde conduit à traiter les informations nociceptives exactement comme dans le cas d'une écoute et d'une vision objectives. L'image et le son cérébraux ressemblent à la perception réelle.

Si les recherches menées grâce aux nouveaux outils de l'imagerie cérébrale ne remontent qu'à la fin des années 1980, le principe d'un vécu corporel lié à l'hypnose est en revanche solidement attesté depuis quarante ans dans l'expérimentation thérapeutique. Du vaste corpus disponible, nous pouvons particulièrement extraire les données cliniques relatives à l'aversive therapy. Son protocole repose sur un conditionnement négatif simple. Pour libérer un patient souffrant d'une dépendance (le tabagisme par exemple), celui-ci est placé sous hypnose et le thérapeute va ancrer une sensation désagréable (le mal de mer) en l'associant au tabac. À partir de ce moment, le fumeur ressentira des nausées, voire pourra être sujet à des vomissements dès qu'il portera une cigarette à ses lèvres ${ }^{16}$. L'aversive training employé en hypnothérapie consiste donc à transformer une action ou une ingestion en un stimulus provoquant une réponse corporelle nocive. La littérature médicale anglo-saxonne (Journal of Nervous and Mental Disease, 1986 ; American Journal of Psychiatry, 1964 ; The British Journal of Psychiatry, 1983; American Journal of Psychoterapy, 1972 ; etc.) témoigne de l'effica-

14. Sur le concept de perceptude en hypnose, voir François Roustang (2003: 179-194). Depuis les travaux « révolutionnaires 》 du psychiatre américain Milton Erickson, le principe de la communication interpersonnelle est devenu la clef de voûte de la cure hypnotique (Bioy et Michaux 2007 : 14].

15. Cette technique d'imagerie permet d'observer l'activité du cerveau à partir des variations locales du débit sanguin. Sur les nouvelles frontières de la «conscience », voir Laureys 2005.

16. Depuis une quinzaine d'années, l'hypnothérapie tente de privilégier des inductions positives pour soigner le tabagisme (Thioly 2007 : 141147). La technique d'aversion reste malgré tout encore utilisée (Bonshtein et al. 2005). 


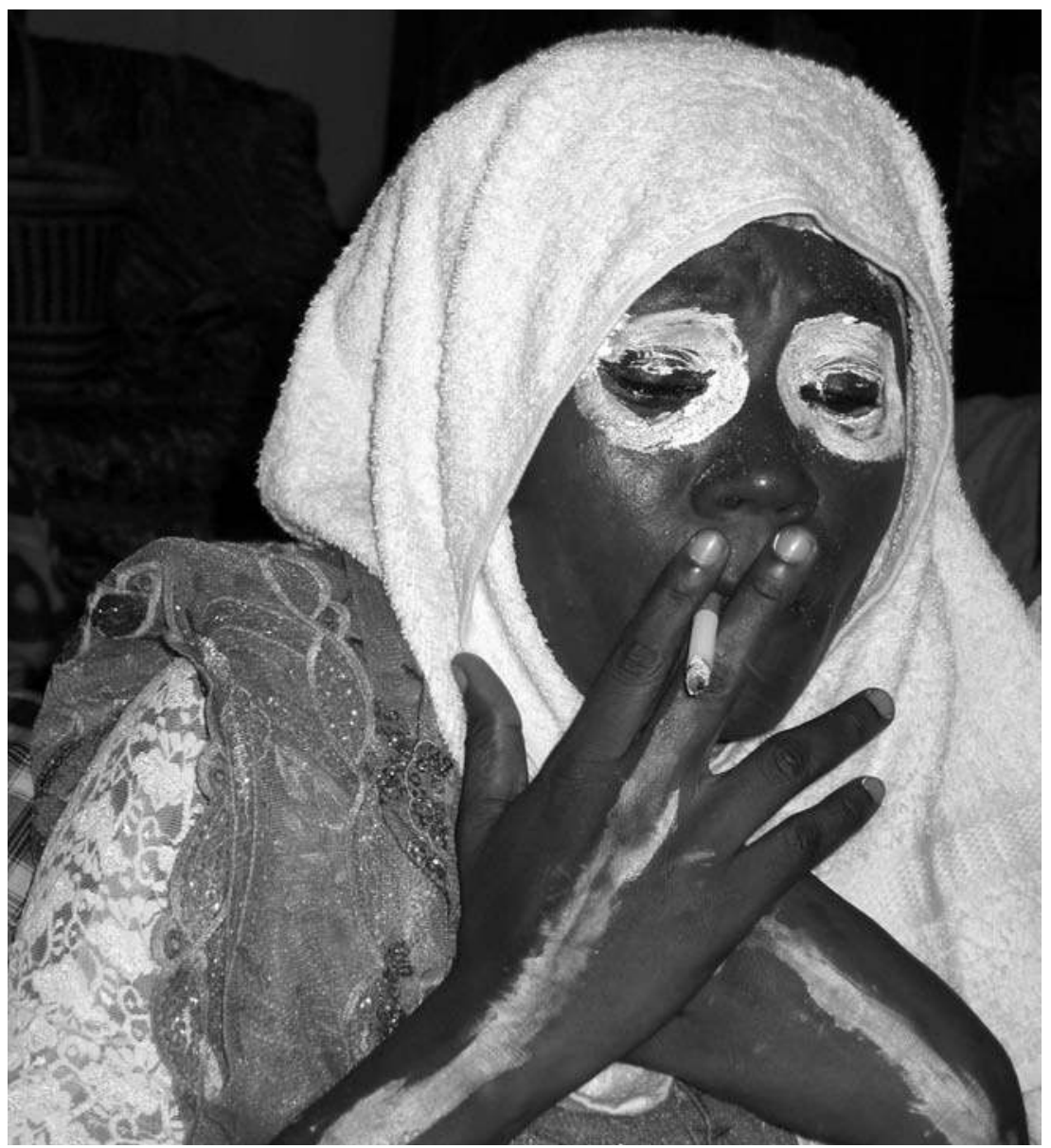

Fig. 8 Possédée par le tromba Mona, Machamou boit de l'alcool et fume. Mayotte, décembre 2006. Photo B. H.

cité de cette technique thérapeutique pour guérir de l'alcoolisme, de la passion compulsive du jeu, de la boulimie ou d'autres syndromes de dépendance.

L'interdit lié à un esprit peut parfaitement jouer cette fonction thérapeutique de réponse répulsive. Ainsi en est-il, par exemple, de la simple vue du bangué (cannabis) pour Zalihata à Mayotte. Cette jeune femme, initiée depuis peu, incorpore le tromba Bevava, qui prohibe strictement l'usage de ce stupéfiant cultivé localement. Or il se trouve que Zalihata fut prise en charge par sa fundi dans un état psychologique grave. Vivant en rupture de ban depuis son adolescence, elle avait mené une vie d'errance qui l'avait conduite à multiplier la prise de psychotropes (datura, cocktail rhum-racines, etc.). Elle fut hospitalisée plusieurs fois à La Réunion pour des crises de bouffées délirantes aigues. Revenue à Mayotte, elle sombrait inexorablement dans la démence quand une parente éloignée, prise de pitié, l'entraîna chez une fundi. Après quelques mois de rumbu-médicaments ponctués de transes très violentes, Bevava s'était manifesté et avait, comme toute première exigence, rappelé son tabou du bangué. Terrorisée, Zalihata respecta scrupuleusement cet interdit durant son initiation. Aujourd'hui, elle est médium attitré, elle a retrouvé son équilibre et mène une vie ordinaire. À ma question sur une éventuelle consommation occasionnelle de bangué, elle répond : «Si j'y touche, je le sais, lui, il va me rendre aussitôt folle. " Ce parcours est aussi celui d'Omar au Maroc. Interné pendant un temps dans un hôpital de Casablanca pour des symptômes de delirium tremens, ce jeune alcoolique fut confié par sa famille, en désespoir de cause, à un chef de culte des Gnawa. Ce dernier identifia en Omar un puissant djinn de la famille des Verts, des esprits musulmans désignés sous le terme « hommes d'Allah » (Hell 2002 : 231). Une fois apparu, ce djinn prohiba formellement tout contact avec l'alcool, n'hésitant pas à provoquer des crises aigues d'étouffement à chaque transgression. Aujourd'hui, Omar est abstinent et il suit « le chemin » ouvert par son djinn.

Forts de ces quelques précisions sur les états hypnoïdes, nous mesurons à présent la corrélation possible entre ces états et le théâtre vécu de la possession. Reste 

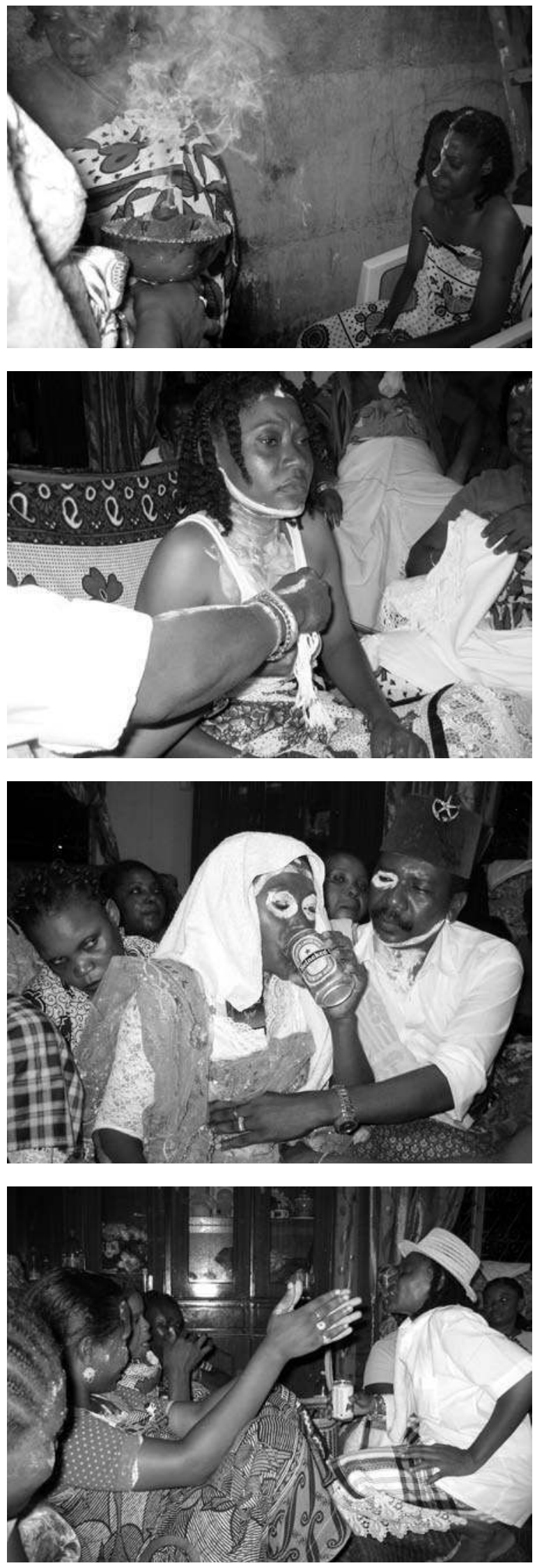

à insister, toutefois, sur un point : à la différence de Georges Lapassade (1997), je ne pose pas l'existence de troubles soit dissociatifs soit hystériques comme fondement effectif de la possession. Même si, à l'évidence, la prise en charge de personnes souffrant d'altérations du comportement constitue un aspect important des cultes, mes références à l'hypnose visent à éclairer plus globalement le processus initiatique qui, lui, ne se limite pas à un dispositif thérapeutique. À mon sens, le puissant vécu corporel et imaginaire des adeptes procède du principe d'ancrage que permet l'induction hypnotique. Une initiation aboutie consiste à inscrire, au plus intime de la personne, à l'état de conscience latente, une (ou plusieurs) personnalité(s) susceptible(s) d'être réactivée(s) à tout moment grâce à certains stimuli volontaires (l'auto-induction de la transe chez les grands initiés) ou inconscients (cas des interdits alimentaires). Mais cette hypothèse ne doit nullement déboucher sur une théorie simpliste du conditionnement, bien au contraire.

\section{Pour une théorie de l'ancrage}

Loin de conduire à un réductionnisme neurobiologique, les avancées des neurosciences montrent que la cognition n'est pas le pur produit d'une logique cérébrale mais qu'elle dépend aussi de constructions sociales et d'expériences personnelles. Les récents travaux sur la plasticité cérébrale en apportent une illustration. Grâce à l'imagerie cérébrale, on constate, par exemple, que les régions spécialisées dans la coordination des mouvements ou dans l'audition peuvent être plus ou moins développées selon que les sujets pratiquent intensément le jonglage ou la musique (Vidal 2005). Bref, en ce qui concerne les mécanismes de conscience et de perception, force est de reconnaître que « la boîte de Pandore ne fait que s'ouvrir" (Faymonville et al. $2005: 49$ ). L'anthropologie de la possession se doit d'intégrer cette complexité du phénomène cognitif. Évoquer le processus d'ancrage lié à une induction hypnotique revient alors à considérer que les notions maintes fois ressassées de "réflexe conditionné » et d' " automatisme » n'épuisent pas le sujet. L'ancrage de l'esprit s'apparente à un véritable travail de la psyché imposant la participation active de l'initié, le but étant de parvenir au

Fig. 9 Avant la venue de ses huit esprits, Machamou est purifiée à l'aide d'encens de copal. Fig. 10 Machamou entre deux possessions. Fig. 11 L'esprit Ndrankenraza aide sa petite-fille Mona à boire en lui appuyant fortement sur le ventre selon un geste rituel lié à l'histoire particulière de cette famille de tromba. Fig. 12 Installé sur Machamou, l'esprit Chianti plaisante avec les spectateurs. Mayotte, décembre 2006. Photos B. H. 
tissage d'une peau de possession. Celle-ci constitue une nouvelle enveloppe de contact avec le monde lui permettant de s'approprier des images signifiantes, de se constituer une mémoire particulière (dont celle des interdits alimentaires) et de construire une interaction sociale fondée sur l'émotionnel (Hell 2006 : 358-360). Dans cette optique, le concept d'ancrage permet de lever le voile sur certaines contradictions apparentes des cultes de possession, en particulier celles inscrites dans le fil de l'opposition authenticité-inauthenticité.

Premièrement, la question de la grande variabilité des formes de la possession traverse de multiples études sans trouver de réponses satisfaisantes. La référence à l'hypnose nous permet ici de ne pas recourir, dans tous les cas, à l'idée de simulation. Les états hypnoïdes peuvent fluctuer considérablement en fonction du contexte et de la nature de l'induction. Ainsi, en ce qui concerne la seule transe profonde, il faut distinguer la " transe somnanbulique " (marquée par une forte activité tonique) et la "transe stuporeuse " (de type cataleptique), lesquelles impliquent des réactions des sujets très différentes (Erickson 1980). Qu'un même tromba puisse provoquer des transes dissemblables chez les initiés, voilà qui ne doit pas non plus surprendre. Et il en est de même de l'inégalité des pouvoirs magico-thérapeutiques que confère l'alliance avec des esprits "guérisseurs » identiques. Car cette " expérience intime », cette puissance de conviction du chamane-thérapeute évoquée par Claude Lévi-Strauss (1958 [1949] : 205) procède directement de la profondeur du processus d'ancrage réalisé durant l'initiation. Les vieux initiés brésiliens, marocains ou mahorais ne disent d'ailleurs rien d'autre lorsqu'ils font remarquer que les esprits ne se manifestent plus avec la même force depuis que les rituels initiatiques se sont raccourcis et édulcorés.

Deuxièmement, le problème des stimuli bénéficie également d'un nouvel éclairage. Les monographies font ressortir la pluralité possible des déclencheurs de la possession au sein d'un même culte. Les possédés peuvent réagir soit à un stimulus sonore, soit à une odeur, soit à une capture sensorielle exercée par le cercle des initiés, soit encore à un simple contact physique. Cette diversité découle du processus d'ancrage effectué. Le maître de l'initiation le module en effet au gré du bricolage interactif qu'il opère avec chaque néophyte. Les ressorts souterrains de la transe requièrent donc une observation ethnographique très précise menée en amont des grandes cérémonies. Observation qui, comme le propose Joël Candau à propos des sensations olfactives, doit aussi intégrer les propriétés pho- nologiques, syntaxiques et sémantiques des mots (2006: 51).

Enfin, au-delà de ces deux points topiques, la théorie de l'ancrage permet d'établir des ponts entre le processus initiatique et le travail psychique mené en hypnoanalyse. Sur ces deux registres, il est fait appel à la force créatrice des rêves, des symboles, des images surgies de la transe, et au ressenti des décharges d'affects. L'imagination active est un levier d'action sur le réel. L'idée qui prédomine est celle d'un inconscient de type resource oriented, selon la formule de Milton Erickson, c'est-à-dire riche de ressources potentielles. Dans cette optique, le thérapeute peut s'appuyer sur les aspects diurnes et nocturnes de l'inconscient du patient pour l'aider à se reconfigurer et à renégocier sa relation aux autres. Suivre ce fil permet alors d'éclairer de nombreux aspects de la possession, tels le principe de la négociation avec les esprits (proche de la « négociation des symptômes " utilisée dans les cures inspirées par Erickson) ou le mécanisme de la relation intersubjective nouée avec le chef de culte (comparable à l'« accordage affectif » connu en hypnothérapie). Ou encore telle, bien sûr, la conception même de la personne prévalant dans les deux systèmes de pensée : une personne dont les limites ne sont pas circonscrites aux frontières de l'identité individuelle mais largement ouvertes aux influences de l'environnement ou de l'histoire transgénérationnelle. Identifier un esprit acquis par héritage ou évoquer la présence morbide d'un " fantôme » familial constituent des diagnostics en étroite résonance.

$\mathrm{Au}$ terme de cet article, une interrogation se fait jour. Le concept de théâtre vécu tel que je le retiens ici reflètet-il encore la pensée de Michel Leiris ? Probablement pas si on l'éclaire à la lumière de la réflexion sartrienne sur la " mauvaise foi » (Jamin 1996 : 40-44). La relecture proposée ne concerne toutefois pas la notion philosophique mais vise à ouvrir, à partir du problème particulier de l'ancrage des interdits, sur une perspective renouvelée du vécu de la possession. Et cette perspective reste, à mon sens, pleinement inscrite dans le champ classique de l'ethnologie française.

mots clés / keywords : cultes de possession // possession cults - Mayotte // Mayotte • interdits alimentaires // food prohibitions • initiation // initiation • contrôle de la transe // control of trance - ancrage hypnotique // hyponotic anchoring.

CEIFR/EHESS

bertrand.hell@univ-fcomte.fr 


\section{Bibliographie}

\section{Althabe, Gérard}

1969 Oppression et libération dans l'imaginaire. Paris, Maspero.

\section{AstuTI, Rita}

2007 « La moralité des conventions. Tabous ancestraux à Madagascar », Terrain 48 : 101-112.

\section{BASTIDE, Roger}

2000 [1958] Le Candomblé de Bahia, rite nagô. Paris, Plon.

1975 Le Sacré sauvage et autres essais. Paris, Payot.

BECKER, Judith

2004 Deep Listeners. Music, Emotion, and Trancing. Bloomington, Indiana University Press.

Bioy, Antoine et Michaux, Didier 2007 Traité d'hypnothérapie. Fondements, méthodes, applications. Paris, Dunod.

BONSHTEIN, Indi, SHAAR, Izhar et Golan, Gabi

2005 « Use of aversion techniques in the hypnotic state for smoking cessation », Contemporary Hypnosis 22(4) : 193-201.

CABRERA, Lydia

2003 La Forêt et les Dieux. Religions afrocubaines et médecine sacrée à Cuba. Paris,

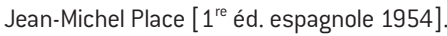

CANDAU, Joë]

2006 «Des odeurs à ne pas regarder... », Terrain 47 : 51-68

\section{CHUCHOTIS}

2006 La Conscience (dossier). Liège, Centre Hospitalier Universitaire.

ClaVerie, Élisabeth

2003 Les Guerres de la Vierge. Une anthropologie des apparitions. Paris, Gallimard.

\section{Coliot, Édouard}

2006 « De la dissociation », in Didier Michaux [dir.], Hypnose et dissociation psychique. Paris, Imago : 105-119.

\section{DeBAEne, Vincent}

2006 «Étudier des états de conscience. La réinvention du terrain par l'ethnologie, 1925-1939 », L'Homme 179 : 7-62.

\section{DiAnTEILl, Erwan}

2000 Des dieux et des signes, initiation, écriture et divination dans les religions afro-cubaines. Paris, École des hautes études en sciences sociales.

\section{ERIBON, Didier}

1988 De près et de loin. Paris, Odile Jacob.

\section{ERICKSON, Milton}

1980 Collected Papers, vol. I. New York, Irvington.

\section{FABRE, Danie]}

1999 « Un rendez-vous manqué. Ernesto De Martino et sa réception en France », L'Homme 151 : 207-236.

FAYMonVILLE, Marie-Élisabeth, Marouet, Pierre et LaUReys, Steven 2005 « Comment l'hypnose agit sur le cerveau », La Recherche 392 : 44-49.

\section{HAMAYON, Roberte}

1995a « Le chamanisme sibérien : réflexion sur un médium », La Recherche, vol. XXVI : 416-422.

$1995 b$ « Pour en finir avec "la transe" et "l'extase" dans l'étude du chamanisme », Études mongoles et sibériennes 26 : 155-190.

\section{HeLl, Bertrand}

1999 Chamanisme et possession. Les Maîtres du désordre. Paris, Flammarion.

2002 Le Tourbillon des génies. Au Maroc avec les gnawa. Paris, Flammarion.

2006 « Possession, émotion et modernité (Maroc, Mayotte) », in Didier Michaux (dir.), Hypnose et dissociation psychique. Paris, Imago : 345-366.

Heusch, Luc de

2006 La Transe, la sorcellerie, l'amour fou, Saint Jean de la Croix, etc. Bruxelles, Complexe.

JAMIN, Jean

1996 «Introduction » à Michel Leiris, Miroirs de l'Afrique. Paris, Gallimard [《Quarto 》) : 9-59.

\section{JAOVELO-DZAO, Robert}

1997 Mythes, rites et transes à Madagascar. Paris, Karthala.

\section{KsENOFONTOV, Gavriil}

1998 Les Chamanes de Sibérie et leur tradition orale. Paris, Albin Michel [ $1^{\text {re }}$ éd. russe 1928].

\section{LAMBEK, Michael}

1993 Knowledge and Pratice in Mayotte: local discourses of Islam, Sorcery and Spirit Possession. Toronto, Toronto University Press. 2002 Weight of the Past. Living with history in Mahajanga, Madagascar. New York, Palgrave Macmillan.

\section{LAPASSADE, Georges}

1997 Les Rites de possession. Paris, Anthropos. Laureys, Steven [éd.]

2005 The Boundaries of consciousness: neurobiology and neuropathology. Burlington Elsevier Publishing Company.

LeIRIS, Michel 1989 [1958] La Possession et ses aspects théâtraux chez les Éthiopiens de Gondar. Montpellier, Fata Morgana [nouvelle éd. augmentée].

1992 C'est-à-dire. Paris, Jean-Michel Place.

LÉvi-STRAuss, Claude

1958 [1949] «Le Sorcier et sa magie», in Anthropologie structurale. Paris, Plon.

1964 Le Cru et le Cuit. Mythologiques I. Paris, Plon.

2003 Entretien avec Dominique-Antoine Grisoni, Magazine Littéraire, hors-série Lévi-Strauss, nº 5 : 16-1?.

\section{MaAndHui, Ousseni}

1996 Parlons shimaore. Mamoudzou, Éditions du Baobab.

\section{MaLinowski, Bronislav}

1933 Trois Essais sur la vie sociale des primitifs. Paris, Payot [ $1^{\mathrm{re}}$ éd. anglaise 1916].

Mauss, Marcel

1950a [1903] «Esquisse d'une théorie générale de la magie », in Sociologie et anthropologie. Paris, PUF.

1950b [1936] «Les techniques du corps », in Sociologie et Anthropologie. Paris, PUF : 363-386.

\section{MétrauX, Alfred}

1958 Le Vaudou haïtien. Paris, Gallimard.

\section{PoIrIER, Jean}

1987 «Tromba et Ambalavelona chez les Bezanozano », in Ethnologiques. Hommages à Marcel Griaule. Paris, Hermann : 277-289.

Pradier, Jean-Marie

2005 « Des chimères de l'abstraction au ravissement des corps en scène », in Internationale de l'imaginaire 20. Arles, Babel-Actes Sud : 113-158.

\section{Rouget, Gilbert}

1990 [1980] La Musique et la Transe. Esquisse d'une théorie générale des relations de la musique et de la possession. Paris, Gallimard. 
2006a Musica reservata. Deux chants initiatiques pour le culte des vôdum du Bénin. Paris, Institut de France, Académie des Beaux-Arts (séance du 26 mars 2005). $2006 b$ « Transe : théâtre, émotions et neurosciences », Cahiers de Musiques traditionnelles : chamanisme et possession 19 : 211-220.

\section{Roustang, François}

2003 II suffit d'un geste. Paris, Odile Jacob.

\section{THioly, Francis}

2007 « TCC et hypnose d'inspiration ericksonienne », in Antoine Bioy et Didier Michaux (dir.), Traité d'hypnothérapie. Paris, Dunod : 119-14?.

\section{Verger, Pierre}

1954 Dieux d'Afrique. Paris, Paul Hartmann. 1982 Orisha. Les dieux yoruba en Afrique et au Nouveau Monde. Paris, Metailié.

\section{VIDAL, Catherine}

2005 Cerveau, sexe et pouvoir. Paris, Belin.

\section{WinkelMAN, Michael}

2000 Shamanism. The neural Ecology of consciousness and Healing. Westport, Bergin and Garvey.

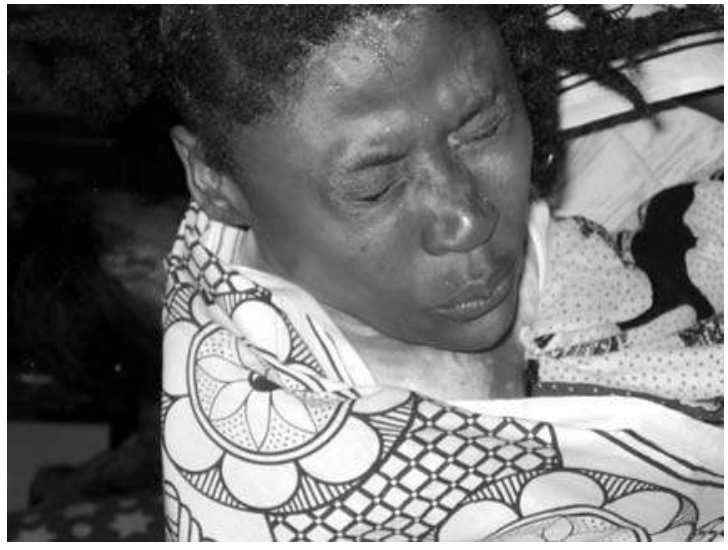

Fig. 13 La montée d'un esprit tromba est toujours douloureuse. Voici le marin Chianti qui s'annonce. Mayotte, décembre 2006. Photo B. H.

\section{Résumé / Abstract}

Bertrand Hell, Négocier avec les esprits tromba à Mayotte. Retour sur le « théâtre vécu 》 de la possession. - À partir de l'observation ethnographique des rituels de fin d'initiation d'une adepte mahoraise du culte des esprits tromba, cet article se propose de revenir sur le concept de «théâtre vécu » introduit par Michel Leiris en 1958. À la même époque, Roger Bastide et Alfred Métraux proposaient, eux aussi, de rompre avec une problématique centrée sur l'opposition authenticité-inauthenticité. Cette voie fut cependant abandonnée par l'ethnologie française, et l'anthropologie de la transe se limita au seul débat entre le structuralisme et le fonctionnalisme. Les récentes avancées des neurosciences relatives à l'hypnose et aux divers états de conscience permettent aujourd'hui de rouvrir le dossier. II est ainsi possible, par exemple, de comprendre le vécu des troubles physiologiques liés à la transgression des interdits qu'imposent les esprits à la lumière de l'aversive therapy utilisée depuis une trentaine d'années par l'hypnose médicale. Plus globalement, une relation est à établir entre le travail initiatique et le processus d'ancrage que permet l'induction hypnotique. Dans une telle perspective, l'anthropologie de la possession peut renouveler ses objets et revisiter utilement ses terrains en s'intéressant, plus en profondeur, aux mécanismes des stimuli, à la diversité des états de possession ou à l'accordage affectif prévalant dans le cercle des initiés.
Bertrand Hell, Negotiating with tromba spirits in Mayotte. The 'théatre vécu' of possession revisited. - Based on ethnographic observation of the rituals ending the initiation of a Mahoré member of the Tromba spirit cult, this article suggests a return to the concept of 'théatre vécu' introduced by Michel Leiris in 1958. During the same period, Roger Bastide and Alfred Métraux also suggested breaking with a paradigm centred on the opposition of authenticity and inauthenticity. However, this route was abandoned by French ethnology, and the anthropology of trance came to revolve around the debate between structuralism and functionalism. Recent advances in neurosciences regarding hypnosis and different states of consciousness allow the theme to be reconsidered. It is now possible, for example, to understand the experience of physiological agitation linked to the transgression of prohibitions imposed by spirits in the light of aversive therapy, which has been used for about thirty years in medical hypnosis. More generally, a relationship can be established between the initiation process and the process of anchoring which permits hypnotic induction. From this perspective, the anthropology of possession can renew its objectives and revisit earlier territory in a useful way by taking a deeper interest in the mechanisms of stimuli, in the diversity of states of possession or in the emotional tuning prevaling within the circle of the initiated 